\title{
Enhanced Power Efficiency of Mobile OFDM Radio using Pre- distortion and Post-compensation
}

\author{
Yuanbin Guo, Joseph R. Cavallaro \\ ECE Department, Rice University, 6100 Main Street, Houston, TX, 77005-1892
}

\begin{abstract}
High power efficiency is an important requirement in cellular transmitter radios. OFDM systems require very linear transmission because of high peak-to-average-power-ratio (PAPR). However, linear power amplifiers (PA) have very low efficiency. To enhance the power efficiency, non-linear PAs are applied and even forced to work near the saturation point. This generates inevitably huge non-linear distortion and spectrum regrowth. In this paper we propose a novel scheme to enhance the power efficiency of the mobile OFDM radio using polynomialbased pre-distortion and post-compensation. We estimate both the non-linearity and inverse non-linearity of the RF transmitter modeled by a memory-less polynomial with Least Square Error (LSE) method. These parameters can then be used to either construct a closed-form pre-distorter or easily generate the postcompensation signal to cancel the non-linear distortion at the RF output.
\end{abstract}

\section{INTRODUCTION}

Also known as orthogonal frequency-division multiplexing (OFDM), multi-carrier modulation (MCM) has been studied extensively because of the robustness in multi-path fading and simplification of equalizer with the application of cyclic prefix (CP) as well as the application of FFT/IFFT for modulation. OFDM has been adopted in several wireless standards such as IEEE 802.11a WLAN and ETSI terrestrial broadcasting.

High power efficiency is an important requirement in cellular transmitter radios. However one major drawback of OFDM is the notoriously high Peak-to-Average Power Ratio (PAPR). Although a linear power amplifier (PA), such as Class $A$, can be applied to provide stable linear transmission, it is not a suitable choice for mobile systems because of the very inefficient use of limited battery power. However if a high efficiency power amplifier is used, serious in-band non-linear distortion as well as adjacent channel interference with spectrum re-growth will occur. To maintain linear transmission, backoff schemes as well as PAPR reduction schemes were studied recently [1,6]. However, the backoff scheme again reduces the power efficiency dramatically and PAPR reduction does not eliminate the requirement of linear transmission completely. Instead, the PA is even forced to work near saturation range to maximize the power efficiency. This will bring inevitably high non-linearity and may outweigh all potential advantages of OFDM. Designing a linear transmitter with high power efficiency is of great importance in mobile OFDM systems.

A complicated pre-distortion technique has been proposed in systems with TWT (traveling wave tube) high power amplifier (HPA) by using the inverse of the non-linearity with Saleh's model and generating the linearly amplified signal at the HPA output $[3,4,5,6]$. Even though, the motivation of the work in this paper is multi-faceted: 1 . Because of the difficulty to get a closed-form solution of the inverse of Saleh's model [3], most previous work used a loop-up-table (LUT)-based Implementation. This has inherent disadvantages with quantization noise caused by the limited size of the LUT and the large size of LUT increasing with the increase of number of bits representing the signal as well as a high time component involved in the fulfillment of the LUT after estimating the property of HPA; 2. Solid-state-power-amplifier (SSPA) is generally used in mobile systems instead of TWT but unfortunately not much work is reported for SSPA in the context of OFDM. 3. The analysis about the PAPR and power efficiency in OFDM is a topic of growing interest.

In this paper we propose a novel parameter-based predistorter and post-compensation to enhance the power efficiency. Both the non-linearity and inverse non-linearity are estimated as memory-less polynomials using LSE criterion. By using closed-form inverse of the non-linearity we constructed a parameter-based pre-distorter in the base-band with fully digital processing for weak non-linearity. In another postcompensation scheme, we achieved linearly amplified output by subtracting the higher order polynomials of the input from the RF in case of deep saturation. The simulations in 16-QAM OFDM show very promising performance.

\section{OFDM SYSTEM MODEL}

In the transmitter of an OFDM system, a set of information bits $\left[\begin{array}{lllll}b_{1} & b_{2} & b_{3} & \ldots & b_{M}\end{array}\right]$ are first mapped into the I/Q channel baseband symbols $\left\{\mathrm{S}_{\mathrm{n}}{ }^{(\mathrm{i}, \mathrm{r})}\right\}$ using a modulation scheme such as phase-shift-keying (PSK) or quadrature-amplitude-modulation (QAM). Then each $\mathrm{N}$ symbols are packed into a parallel block $\left[\begin{array}{llll}S_{1}^{r, i} & S_{2}^{r, i} & \cdots & S_{N}^{r, i}\end{array}\right]^{T}$ at the input of the IFFT. OFDM symbols in the time domain over time interval $t \in\left[0, \mathrm{~T}_{\mathrm{s}}\right]$ are generated by IFFT,

$$
s(k)=s_{r}(k)+j s_{i}(k)=\frac{1}{\sqrt{N}} \sum_{n=1}^{N} S_{n} e^{j 2 \pi(k-1)(n-1) / N}
$$

for $k=[1,2, \ldots, N]$. Then in the so-called Cyclic Prefix (CP) stage, the first $G$ coefficients are repeated after the original $N$ coefficients and made serial for quadrature modulation. We get the analog signal after DAC as,

$$
\tilde{s}(t)=\mathfrak{R}\left\{x(t) e^{j\left(\omega_{c} t+\phi(t)\right.}\right\}
$$

with the amplitude and phase of the input signal to the RF transmitter, 


$$
\begin{aligned}
x(t) & =\sum_{i=0}^{+\infty} \sum_{k=0}^{N_{\tilde{S}}-1} \sqrt{s_{I}^{2}(k)+s_{Q}^{2}(k)} p\left(t-k \frac{T_{\widetilde{S}}}{N_{\widetilde{S}}}-i T_{\widetilde{S}}\right) \\
\phi(t) & =\tan ^{-1}\left(\frac{\widetilde{s}_{Q}(t)}{\widetilde{s}_{I}(t)}\right)
\end{aligned}
$$

with $p(t)$ the pulse function, $T_{\widetilde{s}}$ the period for one symbol and $N_{\tilde{s}}$ the number of samples after CP.

A RF transmitter using super-heterodyne architecture will include several analog components such as passive band-pass filter, passive mixer and power amplifier. Each of these RF components has certain level of non-linearity and can be considered as a non-linear subsystem. The signal in (2) is the input to a cascaded non-linear system of multi-stage analog circuits.

\section{POWER EFFICIENCY VS. NONLINEARITY}

Because of the summation feature of OFDM signal, it can be approximated as a Gaussian distribution according to the Central Limit Theorem. Thus the signal of (2) has a very high PAPR, which is defined as maximum power over the average power of the signal [1]. The high PAPR of OFDM signal requires a very good linear transmission of the signal.

Power amplifiers are typically the most power-hungry components of RF transceivers. The design of PAs, especially for linear, low-voltage operations, remains a difficult problem defying an elegant solution. The class $A$ amplifier operates linearly across the full input and output range. However most class $A$ linear PAs for portable devices at present exhibit efficiencies only around $30 \%$ to $40 \%$ [7]. Although class $C$ PA has a maximum efficiency of $100 \%$, it only exhibits a high efficiency if it delivers a fraction of the peak output power. In a portable transceiver, the efficiency at full output power is of great concern. To achieve higher power efficiency, it is possible to begin with a nonlinear $P A$ and apply linearization techniques to the circuits. Class $E$ PAs are nonlinear amplifiers that achieve efficiencies approaching $100 \%$ while delivering full power, which is a remarkable advantage over class $C$ circuits. Although analog linearization was studied, it requires complicated tuning at analog part and becomes less effective as device characteristics change with the temperature and output power.

Two types of PAs are used for communication systems: TWT in high power satellite communications and SSPA for several other communication systems, especially mobile systems. The complex output of RF with non-linear distortion can be expressed as

$$
\tilde{x}(t)=A[\rho(t)] e^{j\{\varphi(t)+\Phi(\rho(t)\}}
$$

where $\rho(t)$ and $\varphi(t)$ are the amplitude and phase of the input signal. The normalized AM/AM and AM/PM conversion of SSPA can be presented as [5]

$$
\begin{aligned}
& A[\rho(t)]=\rho(t) /\left[1+\left(\rho(t) / A_{\max }\right)^{2 p}\right]^{1 / 2 p} \\
& \Phi[\rho(t)]=0
\end{aligned}
$$

Here $A_{\max }$ is the maximum output amplitude and the parameter $p$ controls the smoothness of the transition from the linear region to the limiting region. In Fig. $1, A_{\max }=1$ with $p=1,3,10$ are depicted. In real world SSPAs, class $A$ corresponds to a large $p$ with a very linear input-output range but also a very flat compression. Non-linear amplifiers with small $p$ have no critical saturation point and the curve is compressed smoothly. Even above the saturation point of linear amplifier, there is a wide range before full saturation.

\section{PROPOSED SCHEMES}

Although (5) gives us a simple model for analysis, we will use a memory-less polynomial to capture the non-linear characteristics of the transmitter, regardless of the SSPA type because the inverse of (5) is not easy to solve. The polynomial is written as,

$$
y(t)=\sum_{i=0}^{P} \alpha_{i} x(t)^{i}=\alpha_{0}+\alpha_{1} x(t)+\sum_{k=2}^{P} \alpha_{k} x(t)^{k}
$$

where $x(t)$ and $y(t)$ are input and output of the nonlinear amplifier respectively. $\alpha_{0}$ represents the DC offset, $\alpha_{1}$ is the linear scalar, $\left\{\alpha_{2}, \ldots, \alpha_{P}\right\}$ contribute to non-linearity in the system and $P$ is the highest order. We will show that this model provides several conveniences in estimation and compensation.

In derivation of the schemes, we will use the discrete formulation by assuming that we already get the samples of both $x(t)$ and $y(t)$ in (6). We also assume that the combinational effect of the thermal noise from the RF circuits is considered as white Gaussian noise. We denote the overall non-linearity for cascade non-linear systems $f_{\mathrm{N}}\left(\ldots f_{2}\left(f_{I}(x)\right) \ldots\right)$ as $f(x)$. The proposed pre-distorter and post-compensation schemes consist of two stages: the estimation stage and the compensation stage for the actual signal.

\section{1. LS estimation of non-linearity}

In the estimation stage, we will send a training sequence with sufficient dynamic range to probe the non-linearity. A feedback of the RF output to the base-band with this sequence is sampled,

$$
y_{n}=f\left(x_{n}\right)+w_{n}=\sum_{i=0}^{P} \alpha_{i} x_{n}^{i}+w_{n}
$$

By defining $N x 1$ signal vectors $Y_{t}=\left[\begin{array}{llll}y_{1} & y_{2} & \cdots & y_{N}\end{array}\right]^{T}$, $X_{t}=\left[\begin{array}{lllll}x_{1} & x_{2} & \cdots & x_{N}\end{array}\right]^{T}$, noise vector $\underline{w}=\left[\begin{array}{llll}w_{1} & w_{2} & \cdots & w_{N}\end{array}\right]^{T} \sim N\left(0, \sigma^{2} \underline{\mathrm{I}}\right)$ and non-linearity coefficients vector $\underline{\boldsymbol{\alpha}}=\left[\begin{array}{llllll}\alpha_{0} & \alpha_{1} & \alpha_{2} & \cdots & \alpha_{P}\end{array}\right]^{T}$, we form a $N \times P$ Vandermonde matrix $\bar{X}_{t}=\left[\begin{array}{lllll}1 & X_{t} & X_{t}^{2} & \cdots & X_{t}^{P}\end{array}\right]$ and write (7) in matrix formulation as,

$$
Y_{t}=\bar{X}_{t} * \underline{\boldsymbol{\alpha}}+\underline{w}
$$




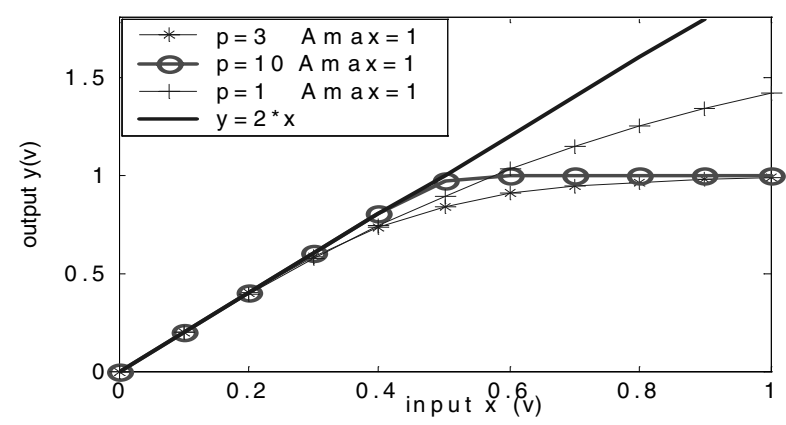

Fig.1. AM/AM response of different SSPAs.

The LS estimation of the coefficients is,

$$
\underline{\hat{\alpha}}=\left[\bar{X}_{t}{ }^{H} \bar{X}_{t}\right]^{-1} \bar{X}_{t}{ }^{H} * Y_{t}
$$

It is known that when the noise is Gaussian, this estimation becomes a minimum variance unbiased estimation (MVUB). Thus we can achieve an optimal estimate of the non-linearity.

\subsection{Pre-distorter}

Several LUT-based pre-distorters can be found in the literature such as Mapping pre-distorter and Gain-based pre-distorter [3]. Although they also estimate the non-linearity characteristics (most of these works used TWT though), the final solution is not parameter-based and has inherent disadvantages as analyzed before. Here we derive a closed-form pre-distorter using a polynomial model.

The purpose of pre-distortion is to find a function $g(x)$ to make the overall effect of RF output linear. By denoting $g(x)$ as another Q-order polynomial $\tilde{x}(n)=g(x(n))=\sum_{i=0}^{Q} \beta_{i} x^{i}(n)$, we expect

$$
\tilde{y}(n)=\sum_{i=0}^{P} \alpha_{i}\left(\sum_{j=0}^{Q} \beta_{j} x^{j}(n)\right)^{i}=\sum_{k=0}^{P * Q} \gamma_{k} x^{k}(n) \approx \alpha_{1} x(n)
$$

with $\gamma_{k}=\xi\left(\alpha_{i}, \beta_{j}\right)$ a function of both $\alpha_{\mathrm{i}}$ and $\beta_{\mathrm{j}}$. The direct solution of this is extremely difficult. Notice that $f^{-l}(y)$ is fitted by another polynomial from training data,

$$
x_{t}(n)=f^{-1}\left(y_{t}(n)\right)=\ell(y)=\sum_{i=0}^{Q} \lambda_{i} y_{t}^{i}(n),
$$

similar to the forward non-linearity, the solution is,

$$
\hat{\Lambda}=\left(\bar{Y}_{t}^{H} \bar{Y}_{t}\right)^{-1} \bar{Y}_{t}^{H} \bullet X_{t}
$$

with $\bar{Y}_{t}=\left[\begin{array}{llll}Y_{t} & Y_{t} & \cdots & Y_{t}^{\varrho}\end{array}\right]$ Vandermonde matrix of the output signal vector and $\hat{\Lambda}=\left[\begin{array}{llll}\hat{\lambda}_{0} & \hat{\lambda}_{1} & \cdots & \hat{\lambda}_{Q}\end{array}\right]$ the estimated inverse non-linearity coefficients. Then the solution of $g(x)$ is

$$
\begin{aligned}
& g(x(n))=f^{-1}(\tilde{y}(n))=f^{-1}\left(\hat{\alpha}_{1} x(n)\right) \\
& \quad=\ell\left(\hat{\alpha}_{1} x(n)\right)=\sum_{i=0}^{Q} \lambda_{i} \hat{\alpha}_{1}^{i} x^{i}(n)=\sum_{i=0}^{Q} \beta_{i} x^{i}(n)
\end{aligned}
$$

with $\left\{\beta_{i}=\lambda_{i} \hat{\alpha}_{1}^{i}\right\}$.

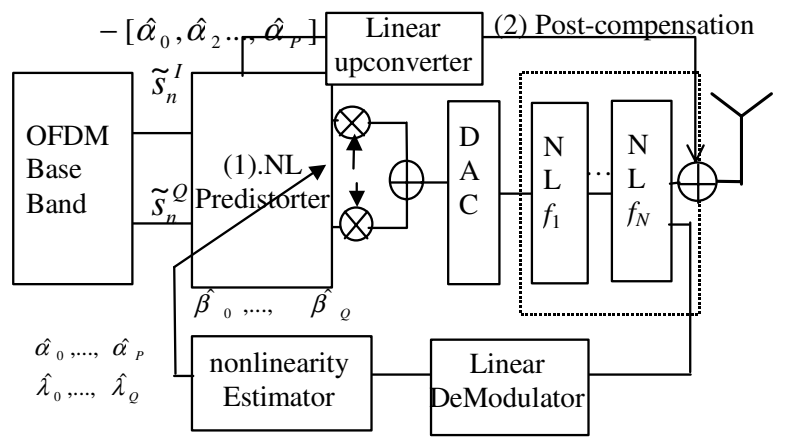

Fig.2. Predistortion and Post-compensation using Polynomials.

\subsection{Post-Compensation}

The compensation of non-linearity from the polynomial model is straight-forward. Since $\alpha_{1}$ is the linear term in (6), a linearly amplified signal can be obtained by subtracting those nonlinear terms when sending actual data as,

$$
\begin{aligned}
\tilde{y}(n) & =y(n)-\sum_{\substack{i=0 \\
i \neq 1}}^{P} \hat{\alpha}_{i} \tilde{x}(n)^{P} \\
& =\alpha_{1} \tilde{x}(n)+\sum_{\substack{i=0 \\
i \neq 1}}^{P}\left(\alpha_{i}-\hat{\alpha}_{i}\right) \tilde{x}(n)^{P}=\alpha_{1} \tilde{x}(n)+\varepsilon(n)
\end{aligned}
$$

The error of this compensation is caused by the estimation error $\mathcal{E}(n)$ and is considerably small. The architecture with both pre-distortion and post-compensation is as Fig. 2. In this architecture, a linear demodulator will sample the RF output and the coefficients are estimated in the DSP base-band. The pre-distortion is constructed as (13) and post-compensation as (14). An overhead of linear up-converter for the postcompensation is required. The output signal is linearly amplified with scalar $\alpha_{1}$. Although post-compensation has the overhead, it can work in deep saturations where the performance of pre-distortion is limited, as explained in the performance analysis. Moreover, because it only needs one estimation, it will have less estimation error than the predistorter if the linear up-converter is adjusted well.

Since the power amplifier property varies with time due to aging, temperature changes, supply voltage variations and the power control, the pre-distortion and post-compensation require adaptive updating. The computation for the polynomial schemes is estimation of both the $f(x)$ and $f^{-1}(x)$ in the form of $\left[\left(X^{H} X\right)^{-1} X^{H}\right]$ of Vandermonde matrix. The computation of it can be simplified by using the structure of Vandermonde matrix. For example, by designing the training data $_{x_{i} x_{j}} \neq 1, \forall i, j \in\left\{\begin{array}{llll}1 & 2 & \cdots & P\end{array}\right\}$,

$$
X^{H * X}=\left(\gamma_{i j}\right)_{P \times P}=\left(1-\left(x_{i} x_{j}\right)^{N}\right) /\left(1-\left(x_{i} x_{j}\right)_{i, j}\right.
$$

Further optimization is possible by applying symmetry structure and advanced linear solutions such as Q-R decomposition. These will be studied in future on implementation.

\section{PERFORMANCE RESULTS}

In the analysis we used the normalized model in (5) with $p=1$, $A_{\max }=2$. In simulation, the actual gain we used is $20 \mathrm{~dB}$. This 
specification is typical for a mobile system. $\mathrm{P}=7$ is used as the polynomial fitting. The responses of both pre-distortion and post-compensation are shown in Fig.3. Both schemes generated a very nearly linear amplified signal at the RF output. However, we also noticed a slight divergence from the linearity for pre-distortion when the input is increased to a critically high level and the output is deeply saturated. In simulation the actual response became compressed. But the post-compensation can still generate a linearly amplified signal even with deep saturation.

\subsection{Bit Error Rate}

The BER curves of both schemes are depicted in Fig.4, together with the non-linearly distorted BER with different "output backoff (OBO)" levels. OBO is defined as the maximum output power (saturation power) over the mean power of the transmitted signal as "OBO $(\mathrm{dB})=10 \log _{10}\left(P_{\max } / P_{\text {avo }}\right)$ ". It determines the operating point of the amplifier. It is found that the pure non-linear distortion caused the huge BER compared with linear transmission at $\mathrm{OBO}=5,7 \mathrm{~dB}$. Even when $\mathrm{OBO}$ is as large as $10 \mathrm{~dB}$, there is a degradation of more than $2 \mathrm{~dB}$ in SNR to achieve BER $10^{-4}$. But with pre-distortion, the BER curve is very close to the linear case with $\mathrm{OBO}=7 \mathrm{~dB}$. However, when OBO is increased to $6,5,4 \mathrm{~dB}$, as we expected from the analysis of response curve, we did find a divergence in the BER. This means that pre-distortion works in a limited input range without very deep saturation. But this is still much better than pure non-linearity at the same OBO level. The limitation is actually the feature of all types of pre-distortion schemes. But in the case of post-compensation, when $\mathrm{OBO}=5 \mathrm{~dB}$, the performance is still close to ideally linear.

\subsection{Total Degradation}

It can also be seen that non-linearity effects can be reduced by very large backoff to make the PA always work in the linear region. But this leads to a loss in power efficiency as well as the coverage range of the transmitter. In this context, a useful performance measure is the total degradation (TD) at a given BER level defined as a function of the OBO:

$$
T D=O B O+E_{b} / N_{o}(O B O)-E_{b} / N_{o}(\text { linear })
$$

where $\mathrm{E}_{b} / \mathrm{N}_{0}(\mathrm{OBO})$ is the required SNR to meet a target BER (e.g. $10^{-4}$ ) at a given $\mathrm{OBO}$, and $\mathrm{E}_{\mathrm{b}} / \mathrm{N}_{0}$ (linear) is the $\mathrm{SNR}$ with a linear amplifier at the same target. The intuitive interpretation of this merit is that increasing $\mathrm{OBO}$ can reduce the $\mathrm{E}_{\mathrm{b}} / \mathrm{N}_{0}$ degradation but also reduce efficiency in using the potential of the PA. On the other hand, reducing OBO increases the efficiency, but also increases the $\mathrm{E}_{\mathrm{b}} / \mathrm{N}_{0}$ degradation. Minimizing TD provides a good tradeoff between output power and non-linear distortion degradation by finding an optimum OBO. Shown in Fig. 5 is the total degradation (TD) vs. OBO. It is obvious that pre-distortion has a much lower minimum TD at a lower OBO compared to pure non-linearity. Without considering the signal level limit of the compensation up-converter, post-compensation will have lower TD at lower OBO than pre-distortion. The practical limitation of it will be considered in other work. It should also be pointed out that in the implementation, post-compensation has an overhead of an additional linear up-converter.

\subsection{Power Efficiency}

Two efficiency measurements are most commonly used. The first one is the drain (or collector) efficiency, i.e., DC supply efficiency. It is a measure of the ability of the PA to convert DC power to RF power as delivered to the antenna, which is defined as $\eta_{D C}=P_{\text {out }} / P_{D C}$, where $\mathrm{P}_{\text {out }}$ is the RF power in watts delivered to the antenna and $\mathrm{P}_{\mathrm{DC}}$ is the power in watts supplied to the power amplifier. Another measurement is the Power Added Efficiency (PAE) which is defined as,

$$
\eta_{P A E}=\frac{P_{o u t}-P_{i n}}{P_{D C}}
$$

where $\mathrm{P}_{\text {out }}$ and $\mathrm{P}_{\mathrm{DC}}$ are the same as the drain efficiency and $\mathrm{P}_{\text {in }}$ is the input signal power to the PA. Obviously, the PAE is a measurement of the signal power added by the PA. Usually, the power efficiency is the function of the output power and the class of power amplifier. As we explained earlier, the efficiency of class A is very low while efficiency of class $E$ is very high. Moreover, for all classes of power amplifiers, the efficiency drops dramatically when the input power level is backed off. However, the relation of the power efficiency and output power usually can not be captured by a closed-form function. It is usually defined by measurements for practical commercial PAs [9]. The power efficiency can also be denoted as a function of the amplitude. If we know the probability distribution function of the amplitude, the expected power efficiency can then be computed as $E[\eta]=\int \eta(r) p_{\Re}(r) d r$, which $p_{\Re}(r)$ is the PDF of a specific signal amplitude. In Fig. 6 we demonstrate the enhanced power efficiency by using pre-distortion and measured power efficiency for each output power level. The PDF function of OFDM signals can be approximated as a Rayleigh distribution. For a specific limited peak power, to avoid large non-linearity, the tail of the PDF above the linear range should be limited also. Thus, for unprocessed non-linear PA (e.g. class C), the probability density will be concentrated in low power levels, resulting in a small average power efficiency. For linear PA(e.g, class A), although the linear range can be similar as pre-distorted, the efficiency function is much smaller than class C. For the processed schemes, we both have a higher saturation point and higher efficiency function in high power level, we achieved a much higher expected efficiency. The particular quantity can be calculated with measurement for particular PAs as explained. 


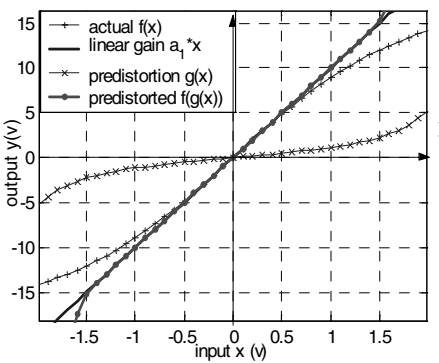

(a). pre-distortion

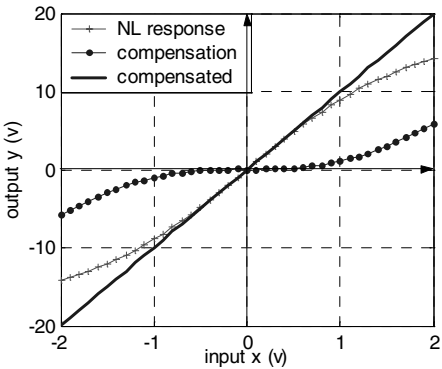

(b) post-compensation

Fig. 3 Responses of the proposed schemes

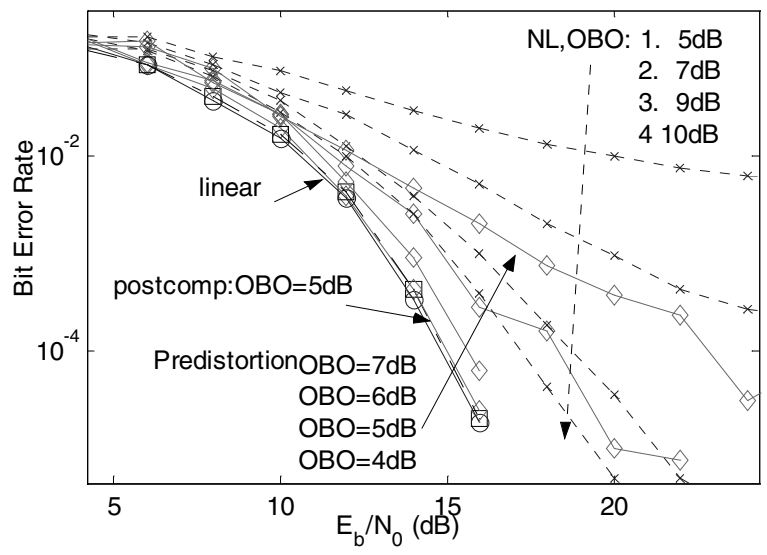

Fig. 4. BER performance with different OBO.

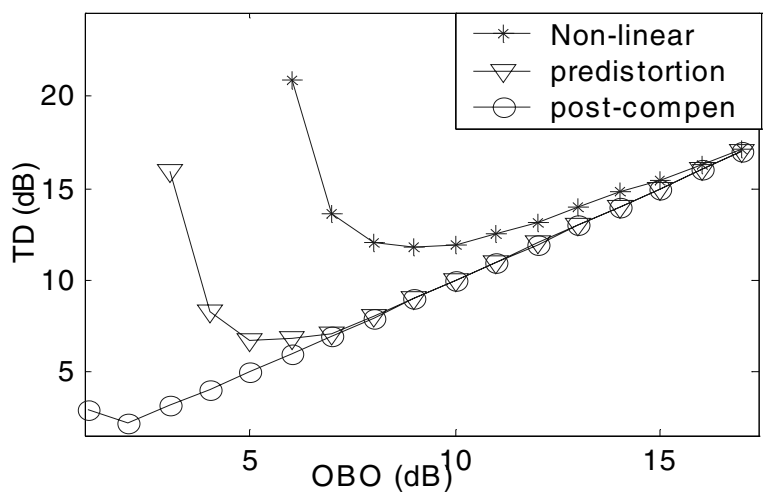

Fig. 5. Total degradation (dB) vs. OBO (dB).

\section{CONCLUSION}

In this paper we derived both a closed-form estimation of the non-linearity and inverse non-linearity of SSPA in a mobile OFDM transmitter using a memory-less polynomial model. Then a fully digital parameter-based pre-distorter as well as a hybrid post-compensation scheme are derived for OFDM. ${ }^{1}$

\section{REFERENCES}

[1] V. Tarokh, H. Jafarkhani, "On the computation and reduction of the peak-to-average power ratio in multicarrier communications", IEEE Trans. on Comm., pp. 37-44, vol. 48, No. 1, Jan. 2000.

\footnotetext{
${ }^{1}$ This work was supported in part by Nokia Corporation, Texas Instruments and the Texas Advanced Program under grant 1999003604-080, and by NSF under grant ANI-9979465.
}

[2] A. Saleh, "Frequency-independent and frequency-dependent non-linear models of TWT amplifiers", IEEE Trans. Comm., Vol. 29, Nov. 1981.

[3] D. Hang, T. Hwang, "An adaptive pre-distorter for the compensation of HPA nonlinearity”, pp. 152-157, IEEE. Trans. on Broadcasting, Vol., 46, No.2, June. 2000.

[4] D. Lottici, V.Reggiannini, "Nonlinear predistortion of OFDM signals over frequency-selective fading channels", IEEE Trans. Comm., pp. 837-843, Vol.49, May2001.

[5] G. Santella, F. Mazzenga, "A hybrid analytical-simulation procedure for performance evaluation in M-QAM-OFDM schemes in presence of nonlinear distortions", IEEE Trans. Vehicular Tech. pp. 142-151, Vol. 47, No.1, February 1998.

[6] J. W. Stark, "Performance analysis of coded multicarrier spreadsprectrum systems in the presence of multipath fading and nonlinearities", IEEE Trans. On Comm. pp. 168-179, Vol. 49, No.1. Jan. 2001.

[7] B. Razavi, "RF microelectronics", 1998 Prentice Hall PTR.

[8] T. Sowlati, Y. Greshishchev, C. A. Salama, "Linear transmitter design using high efficiency class E power amplifier", pp.12331237, 1995.

[9] P. Asbeck G. Hanington, P.F. Chen, "Efficiency and linearity improvement in power amplifiers for wireless communications", pp. 15-18, 1998.

[10] S. Mann, M. Beach, P. Warr, "Increasing talk-time with efficient linear PA's”, IEE Proceeding, pp. 6/1-6/7, 2000.
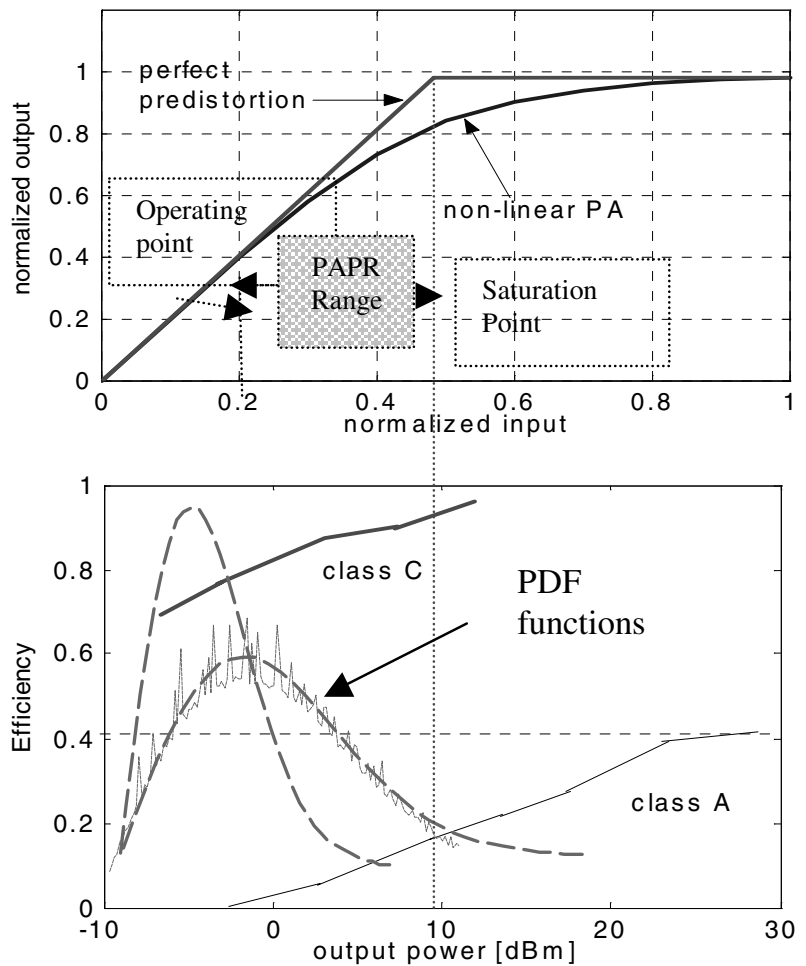

Fig. 6. (a). Clipping response of limited peak-power. (b). Power Efficiency and the probability of different output power level. 